\title{
Using Delay Differential Equations to Induce Alternans in a Model of Cardiac Electrophysiology
}

Justin Eastmana, Julian Sass ${ }^{b}$, Johnny M. Gomesc, Rodrigo Weber dos Santosc, and Elizabeth M. Cherry $\mathrm{d}^{*}$

aDepartment of Mathematics, Millersville University, P.O. Box 1002, Millersville, PA, 17551, USA

bDepartment of Mathematics and Statistics, University of Maryland, Baltimore County, 1000 Hilltop Circle, Baltimore, MD, 21250 USA

${ }^{`}$ Graduate Program on Computational Modeling, Federal University of Juiz de Fora, 36036-900 Juiz de Fora, MG, Brazil

dSchool of Mathematical Sciences, Rochester Institute of Technology, 85 Lomb Memorial Drive, Rochester, NY 14623, USA

* Corresponding author

\begin{abstract}
Cardiac electrical alternans is a period-2 dynamical behavior with alternating long and short action potential durations (APD) that often precedes dangerous arrhythmias associated with cardiac arrest. Despite the importance of alternans, many current ordinary differential equations models of cardiac electrophysiology do not produce alternans, thereby limiting the use of these models for studying the mechanisms that underlie this condition. Because delay differential equations (DDEs) commonly induce complex dynamics in other biological systems, we investigate whether incorporating DDEs can lead to alternans development in cardiac models by studying the Fox et al. canine ventricular action potential model. After suppressing the alternans in the original model, we show that alternans can be obtained by introducing DDEs in the model gating variables, and we
\end{abstract}


quantitatively compare the DDE-induced alternans with the alternans present in the original model. We analyze the behavior of the voltage, currents, and gating variables of the model to study the effects of the delays and to determine how alternans develops in that setting, and we discuss the mathematical and physiological implications of our findings. In future work, we aim to apply our approach to induce alternans in models that do not naturally exhibit such dynamics.

\section{Keywords}

Cardiac arrhythmia mechanisms, Ion channel modeling, Period-doubling

\section{Introduction}

Cardiac alternans is an important dynamical state of the heart characterized by long-short alternations in electrical and/or mechanical properties. Here, we focus on electrical alternans, in which the duration of cardiac cellular action potentials alternate between long and short despite a constant pacing period. Alternans typically appears through a period-doubling bifurcation as the pacing period is decreased. Although alternans may be transient, it is known in both experiments and models to occur as a stable behavior as well. Alternans has been explained as the loss of stability of a fixed point in a one-dimensional map relating action potential duration to the length of the preceding diastolic interval (Guevara et al., 1984; Nolasco and Dahlen, 1968), although a number of limitations of this approach have been noted (Cherry and Fenton, 2004; Cytrynbaum and Keener, 2002; Tolkacheva et al., 2003). Other mechanisms for alternans have been observed as well both experimentally and numerically, including alternans in intracellular calcium cycling (Chudin et al., 1999; Eisner et al., 2000; Restrepo and Karma, 2009; Sato et al., 2013; Shiferaw et al., 2003) and electrotonic coupling between regions with and without conduction block (Bernus et al., 2005; Fenton et al., 2008). 
It has been shown both clinically and experimentally (Gizzi et al., 2013; Pastore et al., 1999) that alternans in the ventricular region of the heart usually degenerates quickly into tachycardia and fibrillation, dangerous arrhythmias that require medical attention. For this reason, alternans has been the target of control strategies (Christini et al., 2006; Garzón et al., 2011, 2009; Hall et al., 1999; Rappel et al., 1999): preventing or terminating alternans, or converting it to less dangerous forms, may be helpful in avoiding some instances of ventricular fibrillation. Alternans also is associated with some atrial arrhythmias (Narayan et al., 2002).

Despite the importance of alternans, many models of cardiac electrophysiology do not display this behavior in their originally published forms, and others may not exhibit realistic alternans in terms of the period at which the bifurcation occurs and the magnitude of alternans (Bueno-Orovio et al., 2008; Cherry and Fenton, 2007; Elshrif and Cherry, 2014). In some cases, modifications of conductance parameters have been suggested to allow the development of alternans, and some phenomenological models have been designed to exhibit alternans based on an analytical connection to restitution (Aliev and Panfilov, 1996; Mitchell and Schaeffer, 2003). However, as cardiac action potential models have increased in complexity from the four-variable Noble model (Noble, 1962) (which includes alternans despite not having a calcium current) to models with dozens of coupled differential equations, how to modify a given model that does not natively display alternans to produce this type of dynamics often is not clear.

In some other fields of mathematical biology, especially population modeling and epidemiology, delay differential equations (DDEs) are used commonly and are known to produce complex dynamics (Bocharov and Rihan, 2000; Smith, 2010). Delays arise naturally in many settings in which sensing or reacting to changes in the system does not occur instantaneously. Some studies (Branco et al., 2010; Burić and Todorović, 2003; Campbell, 2007) have considered the effects of delays in reaction-diffusion systems, including models based on the FitzHugh-Nagumo equations. Recently, it was shown how the introduction of a single delay in the original FitzHugh-Nagumo 
equations can generate mixed-mode oscillations, bursting and chaos (Krupa and Touboul, 2015). In cardiac electrophysiology, delay differential equations have been used previously to describe alternans in a ring geometry (Courtemanche et al., 1996, 1993); more recent work also considered wave propagation in a ring geometry but for more general excitable systems (Gottwald, 2008; Gottwald and Kramer, 2006).

Because of the success of DDEs at introducing complex dynamics into models of other biological systems, we consider here how they can affect the dynamics of models of cardiac electrophysiology. To our knowledge this is the first application of DDEs at the level of ion channel function in individual cardiac cells. For our initial study, we use the Fox et al. model (Fox et al., 2002), which exhibits alternans using the originally published parameter values but which can be modified easily to suppress alternans by changing conductance values or other properties (Fox et al., 2002). We study how replacing one or more ordinary differential equations describing Hodgkin-Huxley-style gating variables from an alternans-suppressed version of the original model with corresponding DDEs can allow alternans to be produced in the model. We explain how the behavior of the gating variables is crucial to understanding when and how alternans can be recreated.

\section{Methods}

To study the effects of delay differential equations in model gating variables on action potential dynamics, we chose to use the Fox et al. model (Fox et al., 2002), which consists of 13 differential equations: the transmembrane voltage $V$, the intracellular calcium concentration, the sarcoplasmic reticulum calcium concentration, and ten gating variables that control the flow of $\mathrm{Na}^{+}, \mathrm{Ca}^{2+}$, and $\mathrm{K}^{+}$ ions across the membrane through ion channels. A total of 13 transmembrane ion currents are summed and determine the rate of change of $V$. The stimulus current used is $80 \mathrm{pA} / \mathrm{pF}$ as used in the original model. The original parameter settings and initial conditions are used unless noted otherwise. 
Each gating variable obeys an ordinary differential equation of the form

$$
\frac{d y}{d t}=\frac{y_{\infty}(V)-y}{\tau_{y}(V)},
$$

where $y$ represents an arbitrary gating variable and $y_{\infty}$ and $\tau_{y}$ its steady-state and time-constant functions, respectively; the only exception is the gating variable $f_{C a}$, for which $\tau_{f C a}$ is a constant and for which $y_{\infty}$ is a function of intracellular calcium concentration rather than voltage. We convert this ordinary differential equation to a delay differential equation (DDE) by evaluating $V$ used in $y_{\infty}(V)$ and $\tau_{y}(V)$ at an earlier time $t-\delta$ for a constant value of $\delta$. In other words, the DDE implementation for a gating variable $y$, with all times made explicit, is

$$
\frac{d y(t)}{d t}=\frac{y_{\infty}(V(t-\delta))-y(t)}{\tau_{y}(V(t-\delta))}
$$

To solve the model equations, we use the Rush-Larsen method (Rush and Larsen, 1978) for the gating variables and the explicit Euler method for all other variables with a fixed time step of $0.02 \mathrm{~ms}$. For the DDE implementation, we restrict the delay $\delta$ to be an integer multiple of the time step, so that we store previous values of $V$ and use the appropriate previous value in calculating $y_{\infty}$ and $\tau_{y}$. Because the beginning of the simulation starts from initial conditions corresponding to pacing at a long cycle length, we use the initial value $V(0)$ for $V(t-\delta)$ whenever $t-\delta<0$.

To assess alternans, we use a downsweep protocol from 310 to $110 \mathrm{~ms}$ in steps of $5 \mathrm{~ms}$ to focus on the range of cycle lengths for which alternans is most likely to occur. At each cycle length, the cell is paced for $10 \mathrm{~s}$ to reach steady state. We measure the action potential durations $(\mathrm{APD}=$ repolarization time - depolarization time) of the last two beats using a threshold of $-80 \mathrm{mV}$ and consider a difference in these APDs of at least $1 \mathrm{~ms}$ to be indicative of alternans. 


\section{Results}

The Fox et al. model as published originally exhibits stable alternans at fast pacing rates, but the alternans can be suppressed through straightforward changes to ion channel conductances (Fox et al., 2002). The availability of both types of behavior allows us to make comparisons between alternans obtained from the ODE and the DDE configurations. Figure 1 compares the original Fox et al. model with the alternans-suppressed version we use most often, in which the conductance $g_{\mathrm{Kr}}$ of the rapid delayed rectifier $\mathrm{K}^{+}$current $I_{\mathrm{Kr}}$ is doubled. Action potentials at a cycle length of $180 \mathrm{~ms}$ for both model configurations are shown in Fig. 1A, with noticeable alternans in action potential shape and duration clearly visible for the original model but absent from the alternans-suppressed version. Fig. 1B demonstrates that alternans occurs for a range of cycle lengths for the original model; for the suppressed model, the action potential duration is decreased over all cycle lengths because of the increased repolarizing current, and alternans does not occur for any cycle length.
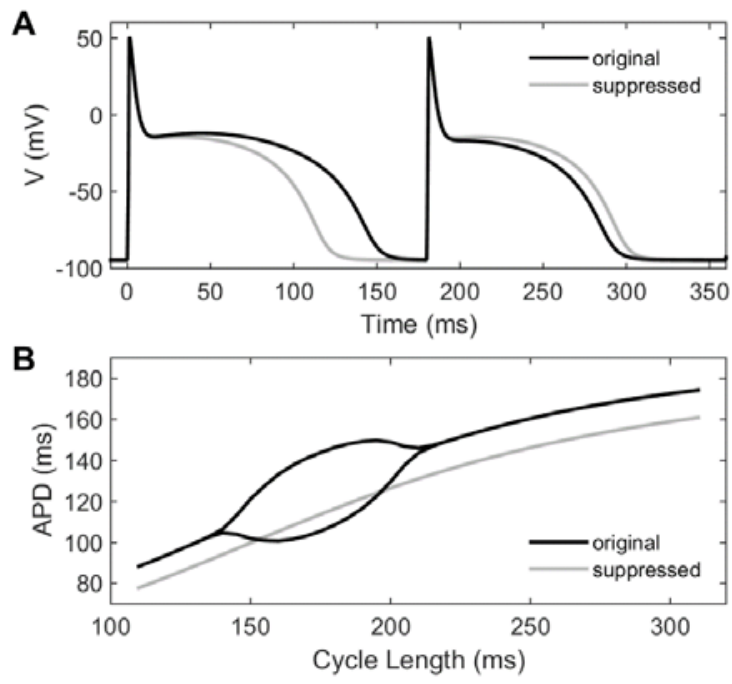

Figure 1. A: Action potentials obtained after pacing the Fox et al. model for $10 \mathrm{~s}$ at a constant cycle length of $180 \mathrm{~ms}$. The original model exhibited alternans with APDs of $146 \mathrm{~ms}$ and $108 \mathrm{~ms}$. To suppress alternans, the conductance $\left(G_{K r}\right)$ of the rapid component of the delayed rectifier $\mathrm{K}^{+}$ 
current was doubled. B: Bifurcation diagram for cycle lengths of 310 to $110 \mathrm{~ms}$ of the original Fox et al. model and of the model with alternans suppressed by doubling $G_{K r}$.

Formulating $I_{\mathrm{Ca}, \mathrm{L}}$ gating variables as DDEs

Once alternans was suppressed by doubling $g_{\mathrm{Kr}}$, we analyzed the effects of introducing delays into each of the ten gating variables present in the model: $d$, the activation gate for the L-type $\mathrm{Ca}^{2+}$ current $I_{\mathrm{CaL}}$; $f$ and $f_{C a}$, the voltage- and calcium-dependent inactivation gates, respectively, for $I_{\mathrm{CaL}}$; $m$, the activation gate for the fast $\mathrm{Na}^{+}$current $I_{\mathrm{Na}} ; h$ and $j$, the inactivation gates for $I_{\mathrm{Na}} ; X_{K r}$ and $X_{K s}$, the activation gates for the rapid and slow components $I_{\mathrm{Kr}}$ and $I_{\mathrm{Ks}}$ of the delayed rectifier $\mathrm{K}^{+}$ current, respectively; and $X_{\text {to }}$ and $Y_{\text {to }}$, the activation and inactivation gates, respectively, of the transient outward $\mathrm{K}^{+}$current. We also introduced a delay in $K_{1}^{\infty}$, the steady-state $I_{\mathrm{K} 1}$ activation function, which in the original model serves as a gating variable for the inward rectifier $\mathrm{K}^{+}$current $I_{\mathrm{K} 1}$ under the assumption that the variable responds instantaneously to changes in voltage.

Delay differential equations applied to any of the gating variables associated with $I_{\text {CaL }}$ could induce alternans. Figure 2 shows the initiation of alternans using a DDE for the $f$ or $d$ gate. For the $f$ gate with delay (Fig. 2A-D), the alternans most closely resembled the original alternans in magnitude for a delay of around $15 \mathrm{~ms}$, although alternans could be induced using a delay as short as $9 \mathrm{~ms}$, as shown in Fig. 3. Nevertheless, the alternans produced did not exactly reproduce the alternans of the original model. In particular, the APD values remained shorter across all cycle lengths because of the increased $I_{\mathrm{Kr}}$ used to suppress alternans before introducing the delay, and the onset and offset of alternans were shifted to longer cycle lengths. The delay in the $f$ gate essentially served to postpone inactivation of $I_{\mathrm{CaL}}$, as shown in Fig. 2C-D. Because inactivation occurred later in the action potential, the current lasted longer when $I_{\mathrm{CaL}}$ was largest, resulting in a larger and longer peak current for the delayed model than for both the original and suppressed models on the first 
peak (long APD); for the second peak (short APD), which was reduced when alternans was present, the peak value with the delay, though still reduced, was near the peak value for the suppressed model and still increased compared to the original model. Note that the increase in $I_{\mathrm{CaL}}$ for the short action potential occurred despite the delay in the recovery of the $f$ gate during repolarization and the diastolic interval. Nevertheless, the delayed decrease in $f$ allowed the current to last longer, thereby broadening its peak.
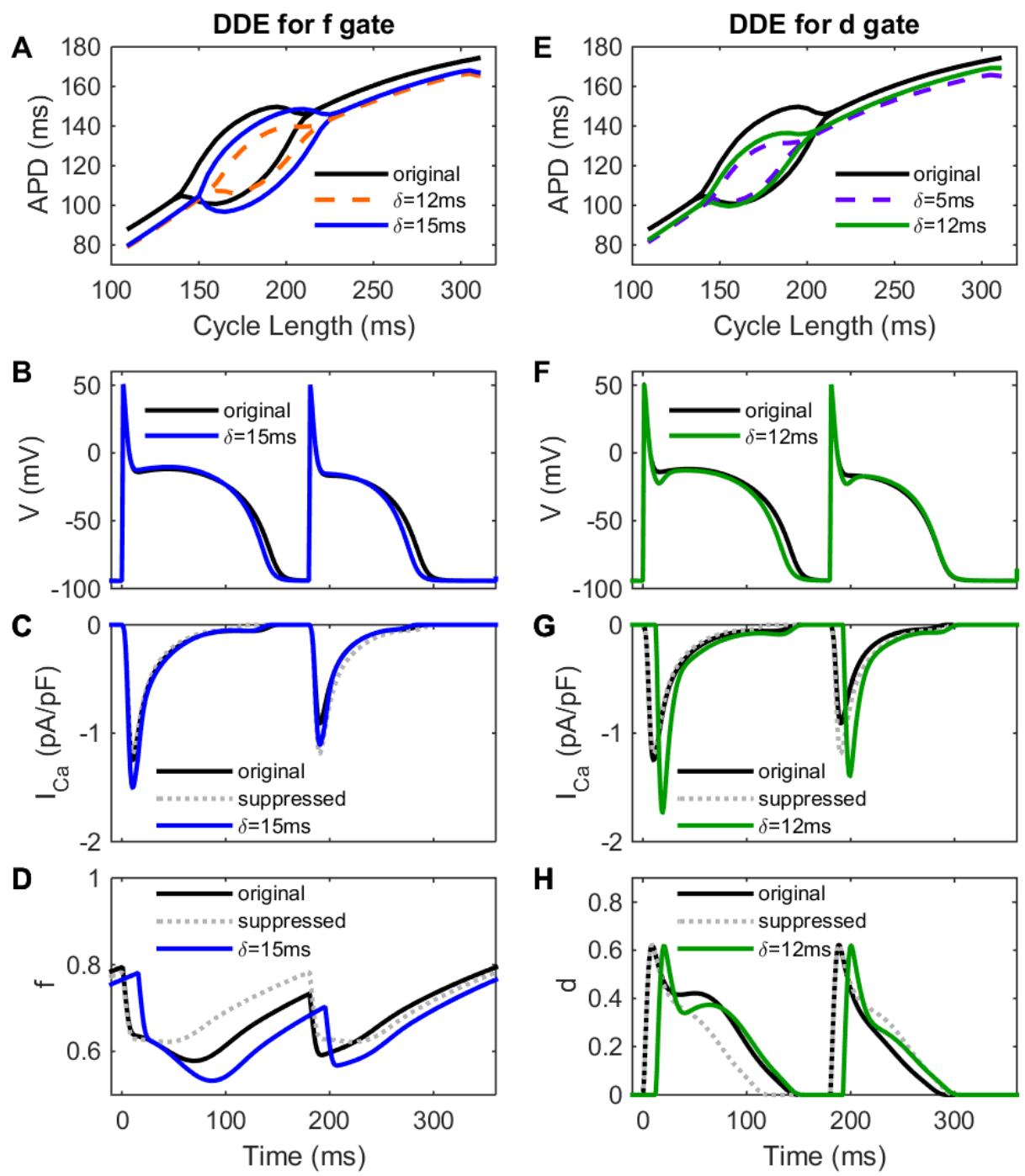

Figure 2. Effects of implementing DDEs for the $f$ and $d$ gates of the L-type Ca ${ }^{2+}$ current $I_{\mathrm{Ca}} \cdot \mathbf{A}$ and $\mathbf{E}$ : Bifurcation diagram for cycle lengths of 310 to 110 ms of the original Fox et al. model and after 
replacing (A) the $f$ gate ODE with a DDE with delays of 12 and $15 \mathrm{~ms}$ and (D) the $d$ gate ODE with a DDE with delays of 5 and $12 \mathrm{~ms}$. B and F: Action potentials obtained after $10 \mathrm{~s}$ of pacing at a cycle length of $180 \mathrm{~ms}$ for the original model and after adding (B) a 15-ms delay to the $f$ gate and (F) a 12-ms delay to the $d$ gate. $\mathbf{C}$ and $\mathbf{G}: I_{\mathrm{Ca}}$ for successive action potentials at a cycle length of $180 \mathrm{~ms}$ for the original, alternans-suppressed, and delayed models. $\mathbf{D}$ and $\mathbf{H}: f$ gate (D) and $d$ gate (F) for successive action potentials at a cycle length of $180 \mathrm{~ms}$ for original, alternans-suppressed, and delayed models.

When a DDE was used to represent the $d$ gate dynamics (see Fig. 2E-H), alternans could be produced using a smaller delay of $4 \mathrm{~ms}$ (see Fig. 3), with a delay of $9 \mathrm{~ms}$ allowing action potentials to resemble those of the original model reasonably closely. However, the action potential shape was more noticeably altered, with changes apparent not only during late repolarization but also during formation of the action potential plateau. The delay in $I_{\mathrm{CaL}}$ activation postponed the development of the current and thus allowed a greater initial repolarization following the action potential upstroke. The development of $I_{\mathrm{CaL}}$ thus was shifted later in the action potential, resulting in morphology changes during plateau development. Delays in $d$ of 5-12 ms resulted in similar cycle lengths for the onset and offset of alternans compared to the original model. 

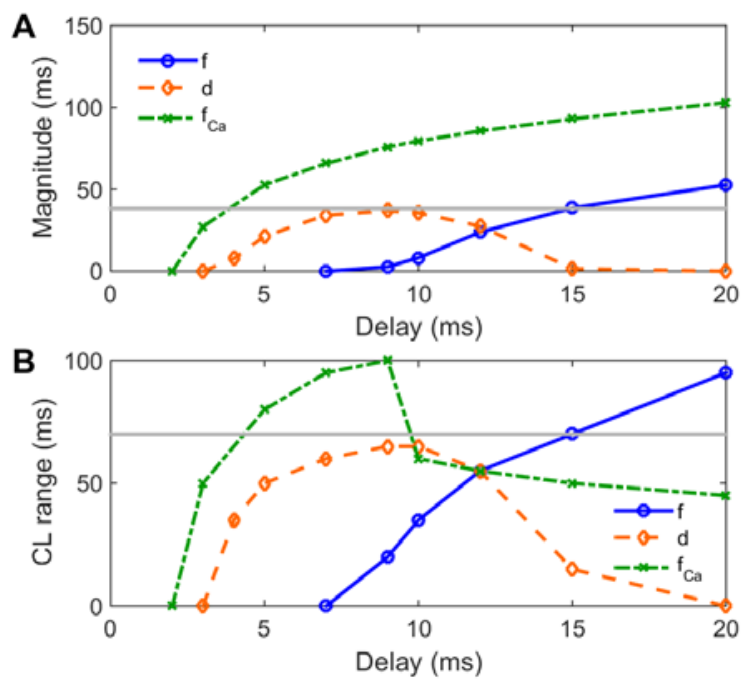

Figure 3. Alternans magnitude (A) and range of cycle lengths exhibiting alternans (B) for delays in the $f, d$, and $f_{C a}$ gates. Gray lines indicate the values for the original model.

A delay to the steady-state value for the $f_{C a}$ gate also could initiate alternans (note that the time constant for this gating variable is a constant in the model, so we did not include any delays in the time constant for this gate, and that the steady-state value is a function of intracellular calcium rather than voltage). Figure 4 shows that the model was more sensitive to delays in this variable and that alternans achieved magnitudes higher than those of the original model by delays as short as $5 \mathrm{~ms}$, with alternans beginning at a delay of $3 \mathrm{~ms}$ and similar to the original model for a delay of $4 \mathrm{~ms}$ (see Fig. 3). Note that the APDs at longer cycle lengths were not reduced nearly so much as when the $d$ and $f$ gates were delayed. Similar to the case of the $f$ gate, the delay here postponed current inactivation and promoted a larger value of the peak current for the long action potential. With $f_{C a}$ delayed, however, the incomplete recovery from inactivation kept the peak current for the short action potential similar to what was seen for the original model. 

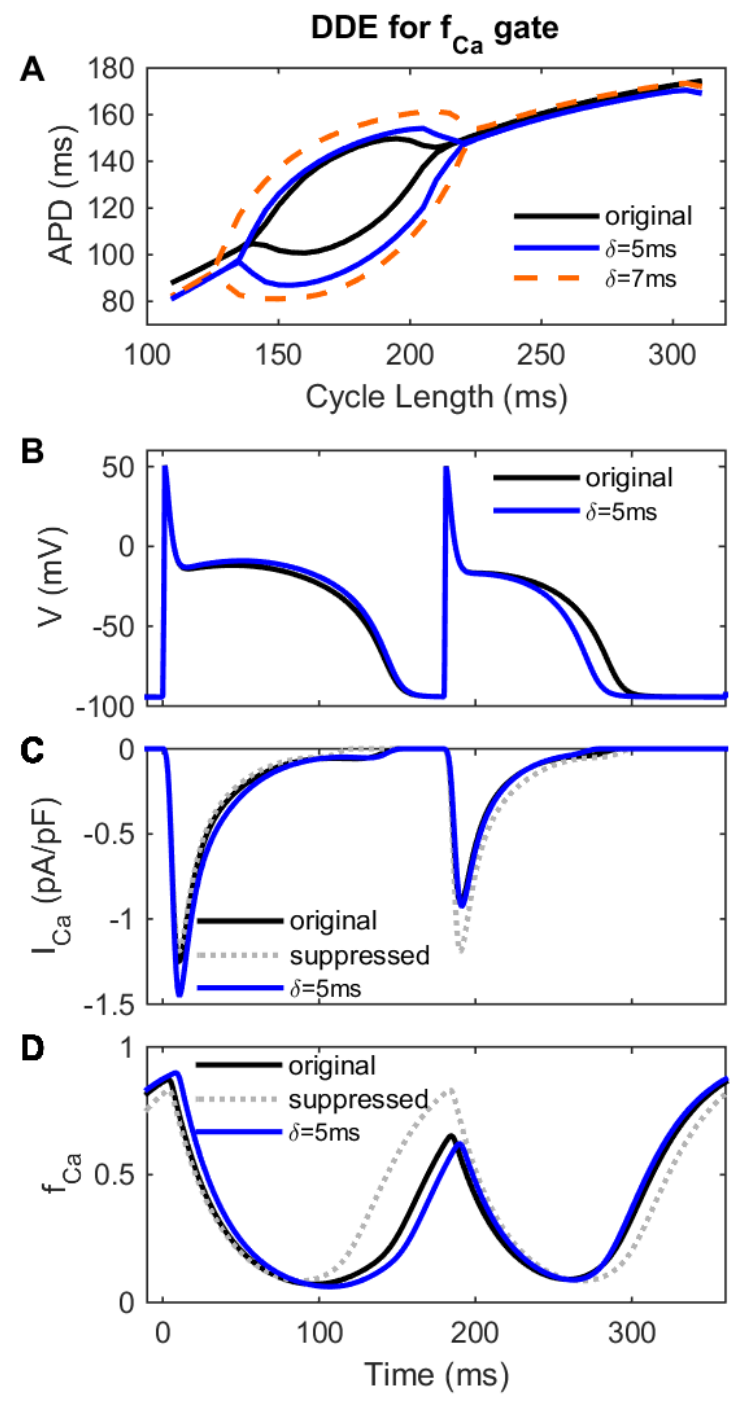

Figure 4. Effects of implementing a DDE in $f_{C a}^{\infty}$ for the $f_{C a}$ gate of $I_{\mathrm{Ca}}$. A: Bifurcation diagram for cycle lengths of 310 to $110 \mathrm{~ms}$ of the original Fox et al. model and after replacing the $f_{C a}^{\infty}$ value in the $f_{C a}$ gate ODE with a DDE with delays of 5 and $7 \mathrm{~ms}$. B: Action potentials obtained after $10 \mathrm{~s}$ of pacing at a cycle length of $180 \mathrm{~ms}$ and after adding a 5-ms delay to the $f_{C a}$ gate and for the original model. C: $I_{\mathrm{Ca}}$ for successive action potentials at a cycle length of $180 \mathrm{~ms}$ for the original, alternanssuppressed, and delayed models. D: $f_{C a}$ gate for successive action potentials at a cycle length of $180 \mathrm{~ms}$ for the original, alternans-suppressed, and delayed models. 
The $I_{\mathrm{Ca}}$ gating variables also could be delayed as a group rather than separately. We tested adding delays to all three possible pairings of two $I_{\mathrm{Ca}}$ gating variables as well as to all three, with the same delay value used in each DDE. Figures 5 and 6 show these results. One of the main findings was that the minimum delay that gave rise to alternans was closely linked to the minimum delay that gave rise to alternans for any of the variables delayed. Thus, because $f_{C a}$ could produce alternans with the smallest delay on its own, its inclusion in any grouping of delayed variables resulted in a similarly small delay value needed for alternans to occur. The one grouping that did not involve $f_{C a}$, that consisting of $f$ and $d$, required a slightly higher delay to initiate alternans. In that case, the delay needed corresponded to that required for $d(4 \mathrm{~ms})$, which required a smaller delay on its own to produce alternans than $f$ did. 

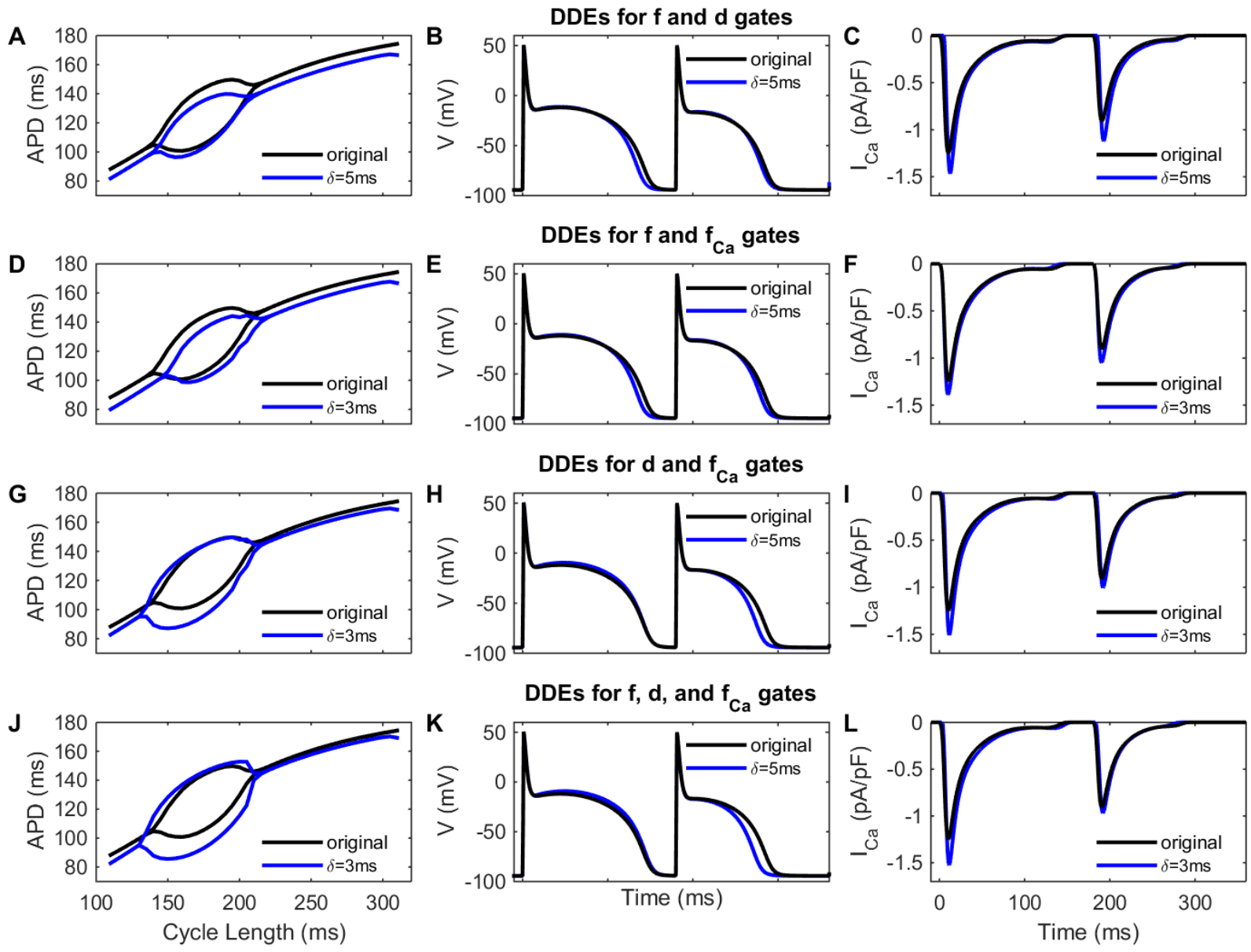

Figure 5. Effects of implementing DDEs simultaneously for combinations of the $d, f$, and $f_{C a}$ gates of $I_{\mathrm{Ca}}$. Bifurcation diagram, action potentials after pacing for $10 \mathrm{~s}$, and $I_{C a}$ after pacing for $10 \mathrm{~s}$ for the original Fox et al. model and (A-C) after replacing the ODEs for the $f$ and $d$ gates with DDEs with a delay of $5 \mathrm{~ms}$, (D-F) after replacing the ODEs for the $f$ and $f_{C a}$ gates with DDEs with a delay of $3 \mathrm{~ms}$, (G-I) after replacing the ODEs for the $d$ and $f_{C a}$ gates with DDEs with a delay of $3 \mathrm{~ms}$, and (J-L) after replacing the ODEs for the $f, d$, and $f_{C a}$ gates with DDEs with a delay of $3 \mathrm{~ms}$. 

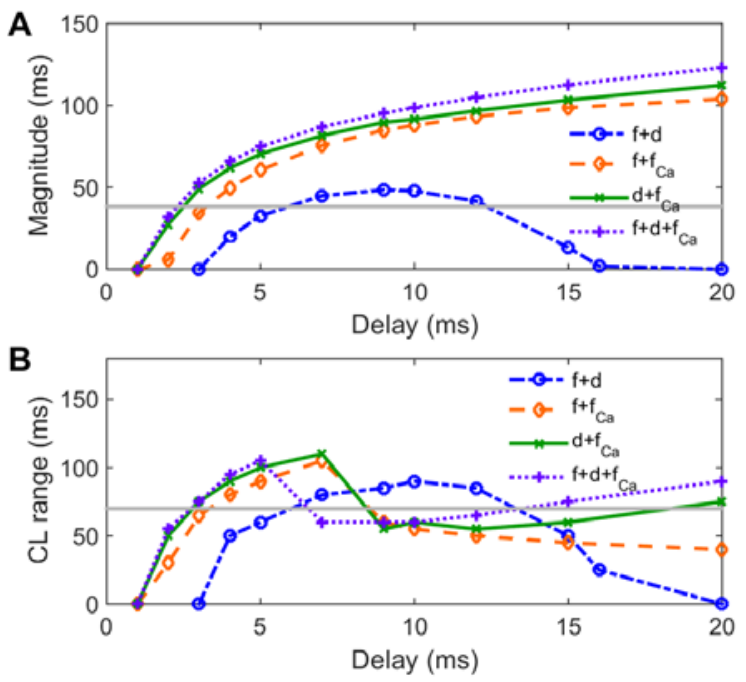

Figure 6. Alternans magnitude (A) and range of cycle lengths exhibiting alternans (B) for delays in combinations of the $f, d$, and $f_{C a}$ gates. Gray lines indicate the values for the original model.

Formulating $K_{1}^{\infty}$ using a DDE

Adding a delay to the equation for $K_{1}^{\infty}$ by evaluating the voltage at a previous time also allowed alternans to develop, as shown in Fig. 7. This variable, which serves as a gating variable for $I_{\mathrm{K} 1}$ under the assumption in the original model that it immediately reaches steady state, was the most sensitive of the gates that could produce alternans; i.e., alternans arose from the smallest delays. A delay on the order of $1.3 \mathrm{~ms}$ allowed alternans to develop, significantly smaller than required for any other variable. However, alternans also disappeared at a small delay value of $2.5 \mathrm{~ms}$, so that it was present over a very small range of delays. The small delays involved likely arise from the fact that in the original model the response to a change in voltage is instantaneous, so that introducing even a small delay can have large effects. In particular, a delay of even a few ms can affect the development of $I_{\mathrm{K} 1}$ in response to the upstroke noticeably, as seen in Fig. 7. 

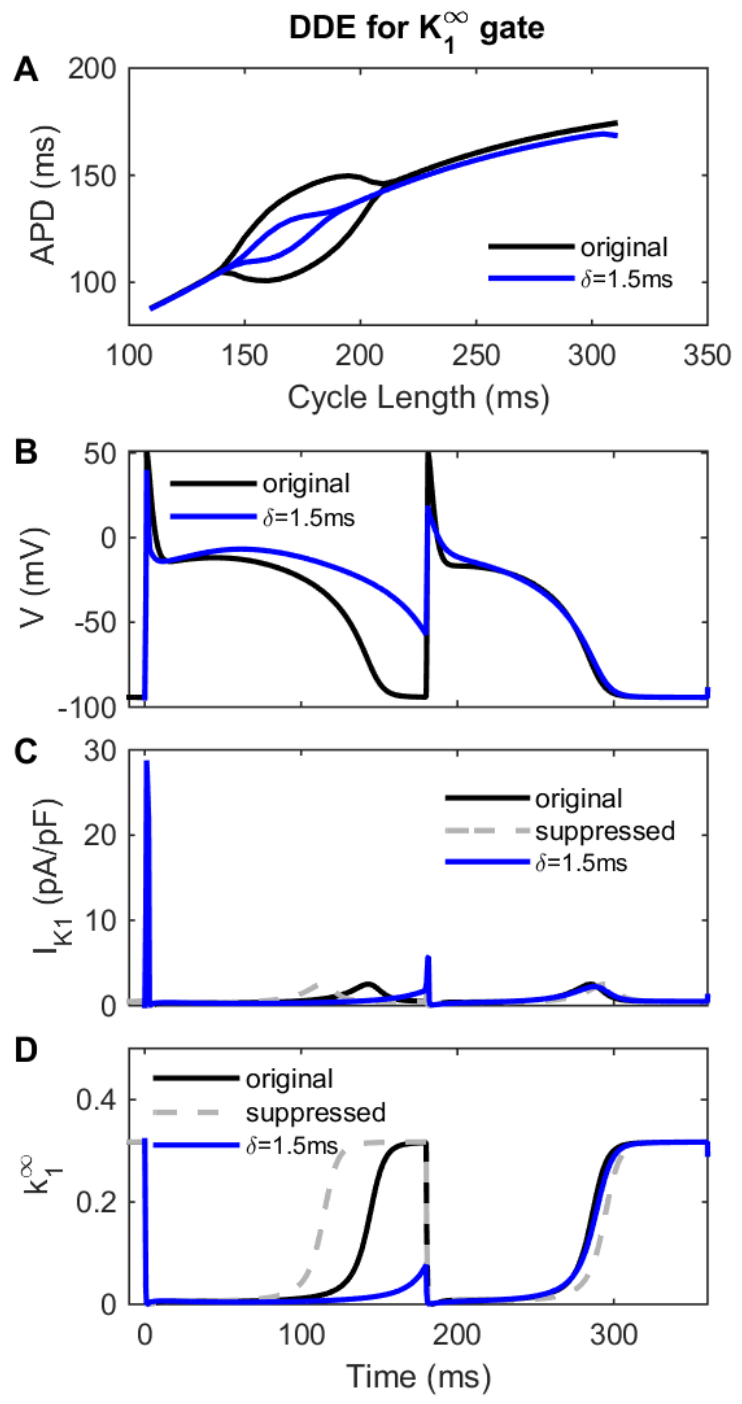

Figure 7. Effects of implementing a DDE in the $K_{1}^{\infty}$ gate of the inward rectifier $\mathrm{K}^{+}$current $I_{\mathrm{K} 1}$.

A: Bifurcation diagram for cycle lengths of 310 to $110 \mathrm{~ms}$ of the original Fox et al. model and after introducing a delay of $1.5 \mathrm{~ms}$ to $K_{1}^{\infty}$. B: Action potentials obtained after $10 \mathrm{~s}$ of pacing at a cycle length of $180 \mathrm{~ms}$ and after adding a 1.5 -ms delay to $K_{1}^{\infty}$ and for the original model. C: $I_{\mathrm{K} 1}$ for successive action potentials at a cycle length of $180 \mathrm{~ms}$ for the original, alternans-suppressed, and delayed models. D: $K_{1}^{\infty}$ for successive action potentials at a cycle length of $180 \mathrm{~ms}$ for the original, alternans-suppressed, and delayed models. 
Figure 8 compares the alternans magnitude and cycle length range (difference between maximum and minimum cycle lengths producing alternans) for delays in all the gating variables considered and shows that the responses of the gates to delays differed significantly. $K_{1}^{\infty}$ showed the greatest sensitivity, in that the smallest delay values could be used.
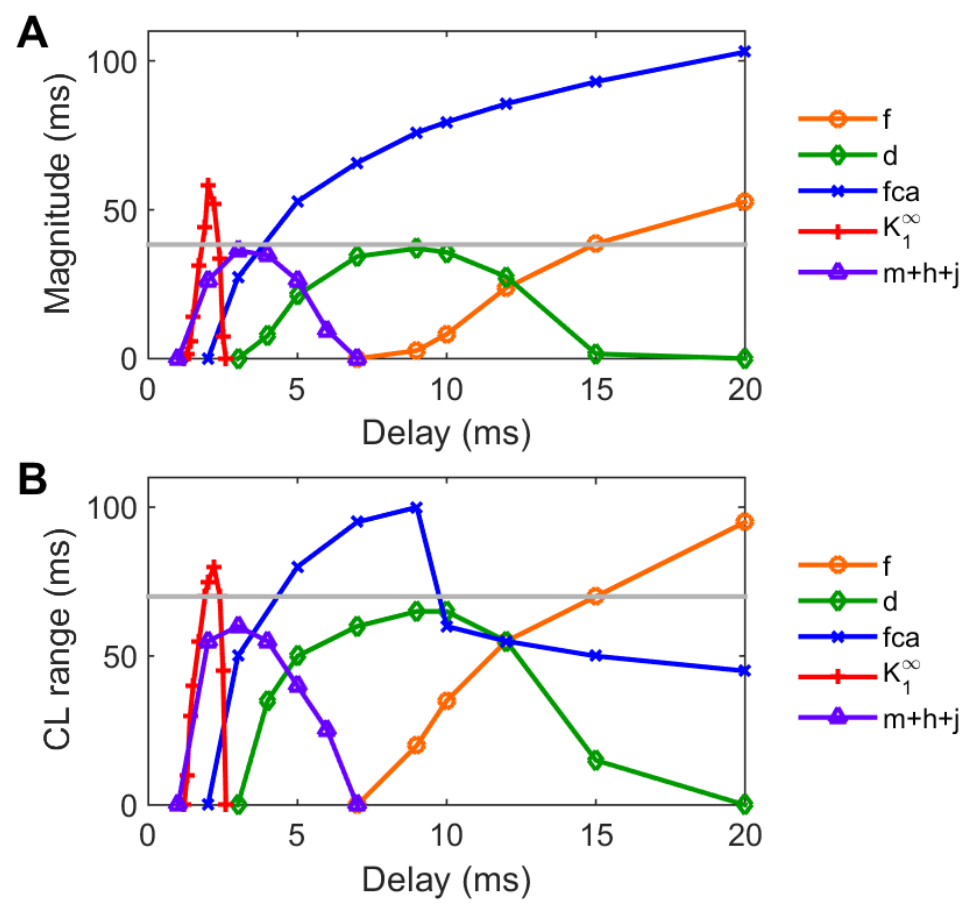

Figure 8. Alternans magnitude (A) and range of cycle lengths exhibiting alternans (B) for delays in the $f, d, f_{C a}$, and $K_{1}^{\infty}$ gates along with the combination of the $m, h$, and $j$ gates. Gray lines indicate the values for the original model.

DDE formulations for other gating variables

Not all gating variables could be used to initiate alternans by converting ODEs to DDEs. The remaining gating variables, $X_{K r}, X_{K s}, X_{t o}, Y_{t o}, m, h$, and $j$, did not produce alternans when DDEs were substituted for ODEs for delays up to 20 ms. As shown in Fig. 9, delays for these gating variables associated with $\mathrm{K}^{+}$currents all resulted in significant action potential shortening (the 
activation variables did so by delaying the inhibition of repolarizing current, while the inactivation variable did so by inhibiting development of the action potential notch and thus reducing $I_{C a}$ ). For the Fox et al. model, modifications causing severe action potential shortening, which led to longer diastolic intervals and more complete recovery of the gating variables, were never observed to allow alternans to occur. Delays applied to the inactivation gating variables associated with $I_{N a}, h$ and $j$, also shortened the action potential duration, with $h$ producing shortening preferentially at longer cycle lengths. Delays applied to the $m$ gate interfered with action potential formation by reducing overall current, thereby preventing action potentials from occurring at all.
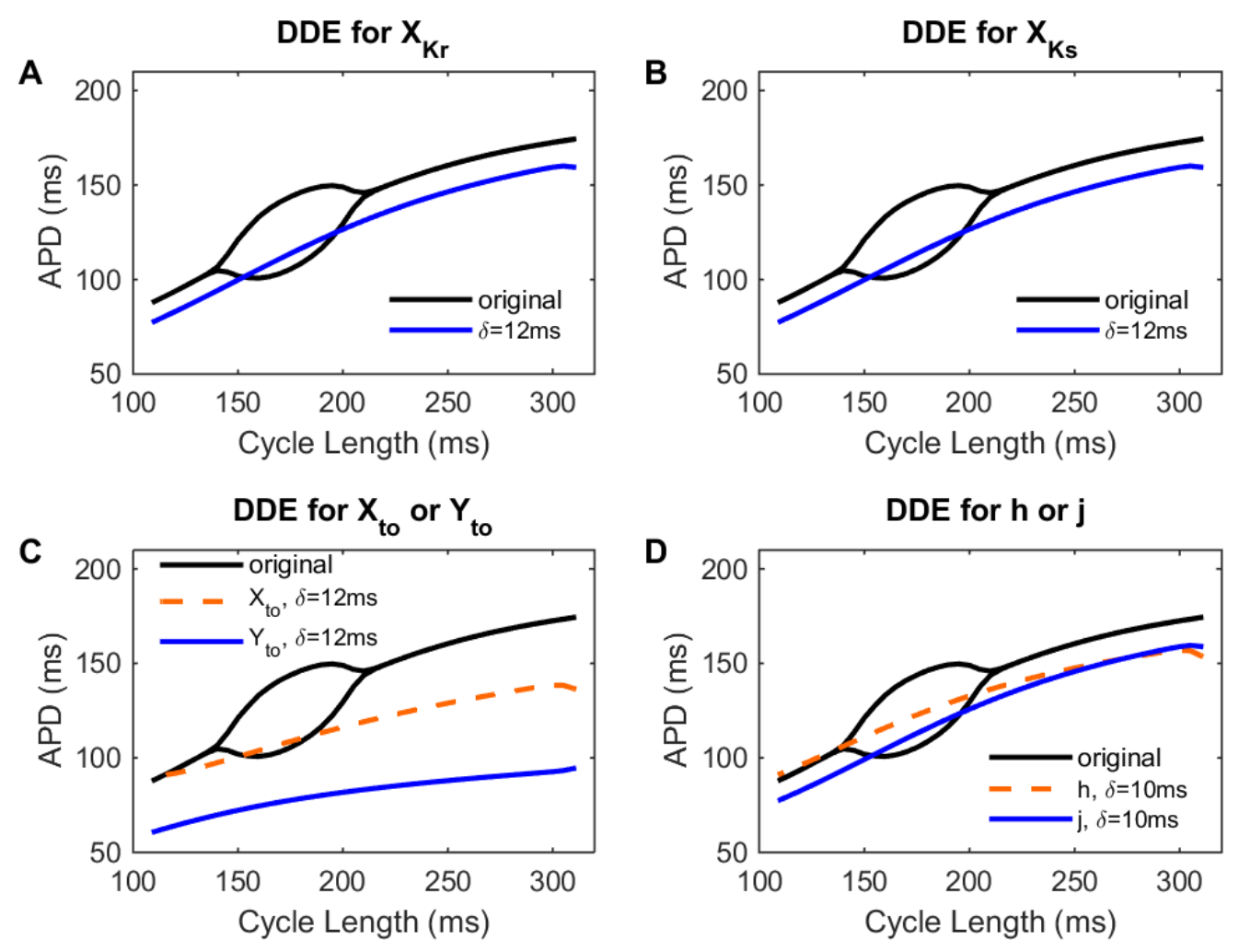

Figure 9. Inability to produce alternans through replacement of ODEs with DDEs for (A) $X_{K r}$, (B) $X_{K s}$, (C) $X_{\text {to }}$ or $Y_{t o}$ and (D) $h$ or $j$. 
Separating delays for time constants and steady-state values

In all cases discussed so far, delays were added to the voltages used in the equations for both the time constants and steady-state values of the gating variables (except for $f_{C a}$ and $K_{1}^{\infty}$, as discussed earlier), but we also considered implementing delays separately. We note that both functions would need to be delayed to ensure that the effects on the underlying forward and reverse voltagedependent rate constants would be consistent when the gating variable equations adhered strictly to the Hodgkin-Huxley formulation. However, many more recent models specify only the steadystate and time constant functions, and some models even use the formulation involving rate constants to determine the time constant but use unrelated equations for the steady-state values. In the Fox et al. model, the gating variables $m, h, j, X_{t o}$, and $Y_{\text {to }}$ are presented using the rate-constantbased formulations, but $X_{K r}, X_{K s}, d, f$, and $f_{C a}$ are not. Therefore, we chose to consider separate delays for one of these gating variables. Figure 10 shows the results of using a delayed voltage value in $\tau_{f}$ but not $f_{\infty}$ and for $f_{\infty}$ but not $\tau_{f}$. In both cases, the minimum delay value needed to induce alternans was reduced compared to when both values were delayed together, with a greater reduction when delaying only $f_{\infty}$ compared to when delaying only $\tau_{f}$. Earlier induction also was associated with increased alternans magnitude and cycle length range. Delaying $\tau_{f}$ or $f_{\infty}$ separately promoted alternans formation by exacerbating changes in the gating variable $f$; changing the two in tandem had a moderating effect on the response of $f$. 

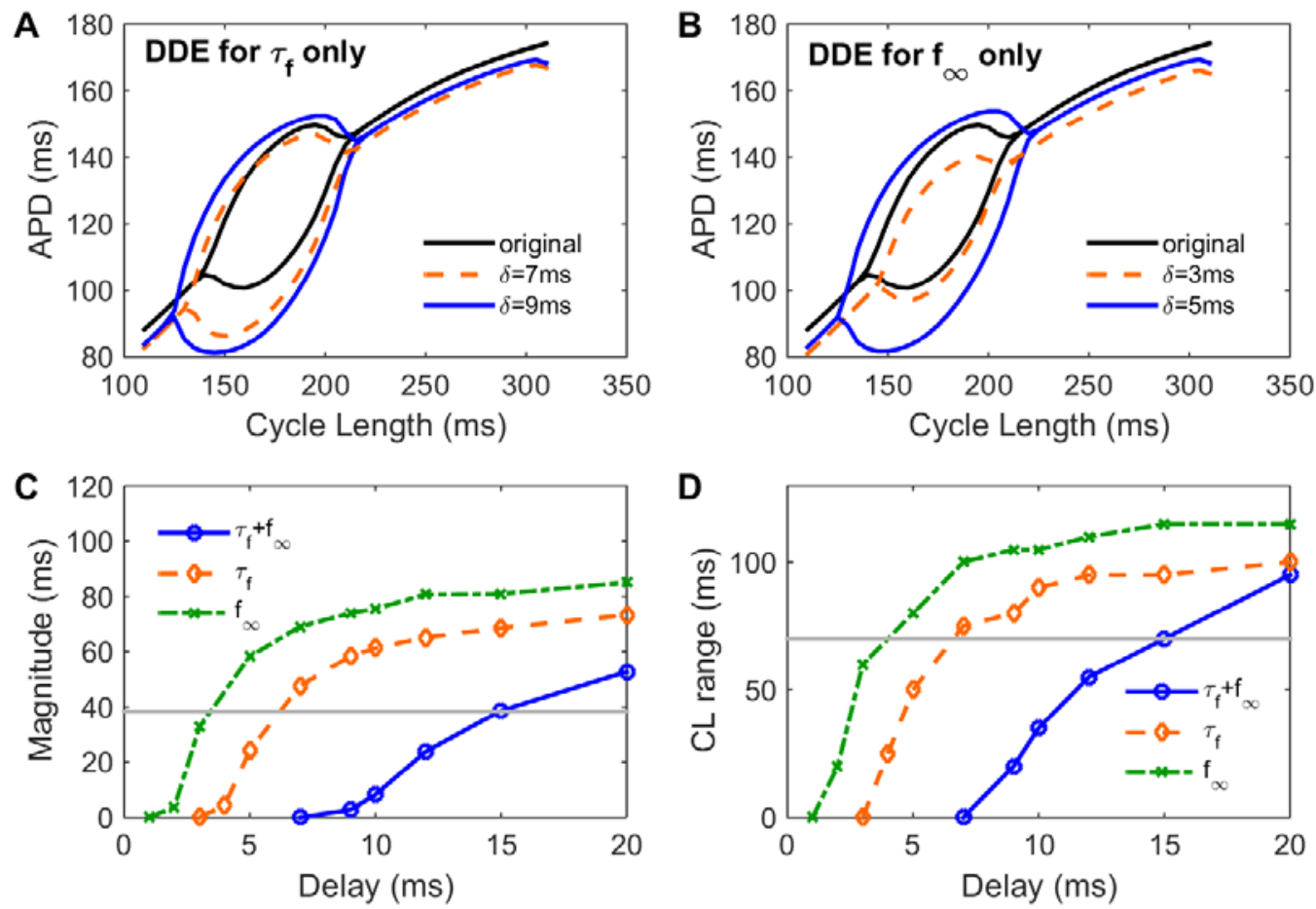

Figure 10. Separating the effects of delays in $\tau_{f}$ and $f_{\infty}$ promoted alternans development for smaller delays. When delays were introduced (A) only to $\tau_{f}$ or (B) only to $f_{\infty}$, alternans could be produced for smaller delays than when both $\tau_{f}$ and $f_{\infty}$ were delayed together. Alternans magnitude (C) and range of cycle lengths exhibiting alternans (D) also varied for separate delays in $\tau_{f}$ and $f_{\infty}$; gray lines indicate the values for the original model.

Other methods for suppressing alternans in the original model

For all the results shown so far, alternans in the original model was suppressed by increasing $G_{K r}$ by a factor of two. However, the successful initiation of alternans did not depend on using this particular approach to suppress alternans. Figure 11 shows six other maximum channel conductance modifications that also suppressed alternans. In each case, it was possible to initiate alternans using a DDE for the $f$ gate, although the magnitude of the delay needed varied among the different methods. Also note that in some cases significant conductance increases were needed 
because the currents involved were small in magnitude, so that larger conductance changes were needed to produce changes in transmembrane current of similar magnitude.
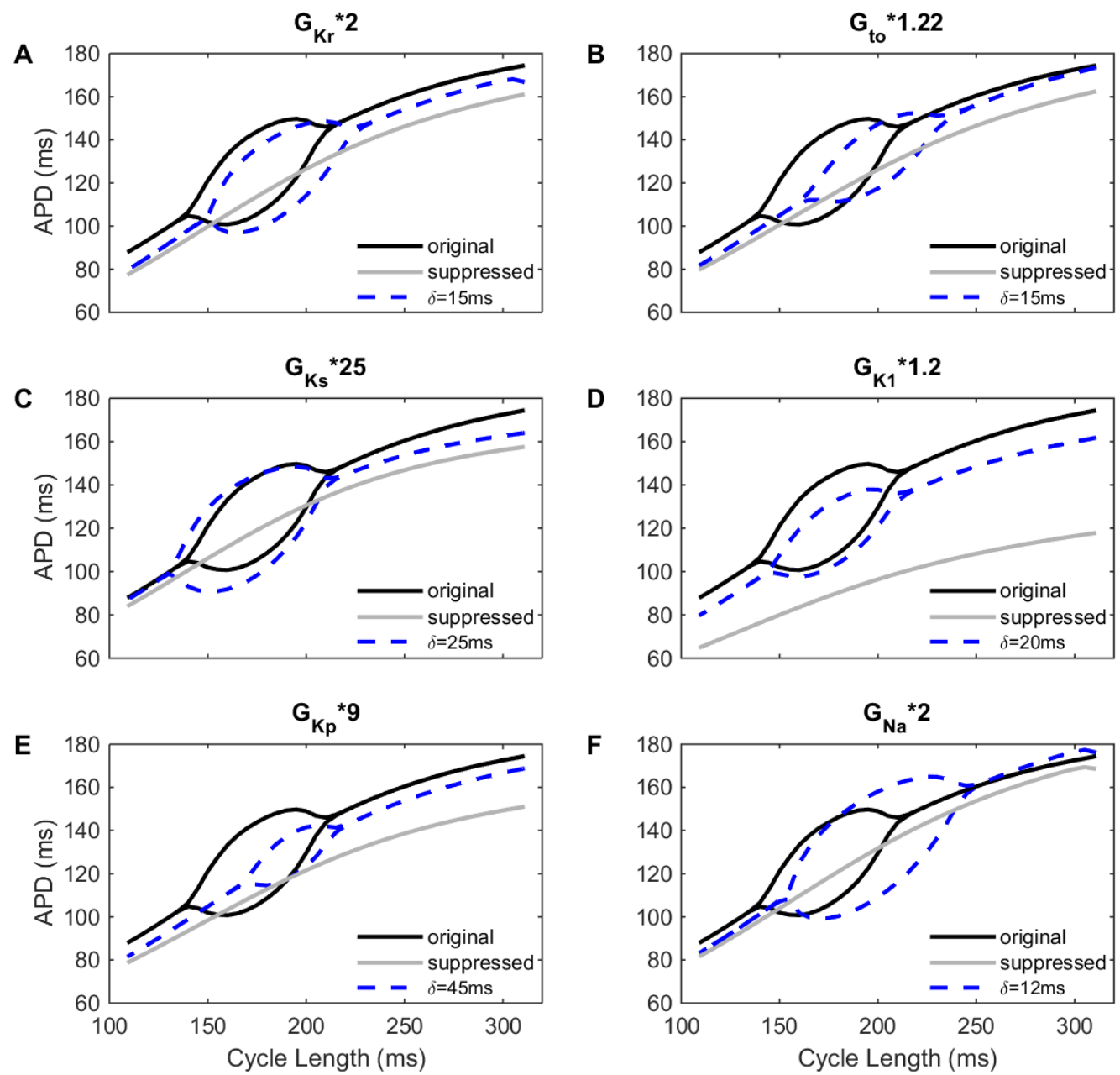

Figure 11. Ability of delays to produce alternans for different alternans suppression methods.

Alternans could be produced with a delay in the $f$ gate using various approaches to suppress alternans, including (A) increasing $G_{K r}$ by a factor of 2, (B) increasing $G_{t o}$ by a factor of 1.22 , (C) increasing $G_{K s}$ by a factor of 25, (D) increasing $G_{K 1}$ by a factor of 1.2 , (E) increasing $G_{K p}$ by a factor of 9 , and (F) increasing $G_{N a}$ by a factor of 2 . Note that larger increases in channel conductances were needed for currents with smaller magnitudes, such as $I_{\mathrm{Ks}}$ and $I_{\mathrm{Kp}}$. 


\section{Discussion}

In this paper, we have presented, to the best of our knowledge, the first use of delay differential equations in transmembrane current gating variables of cardiac cell models. We showed that it is possible to use delay differential equations for gating variables associated with $I_{\mathrm{Ca}}$ and $I_{\mathrm{K} 1}$ to produce alternans in a model where alternans had been suppressed. For this model, the postponement of $I_{\mathrm{Ca}}$ inactivation through delays applied to the inactivation gates had the largest effects, with maximum alternans magnitudes of 53 and $103 \mathrm{~ms}$ for delays up to $20 \mathrm{~ms}$ for $f$ and $f_{C a}$, respectively, and alternans CL ranges up to $100 \mathrm{~ms}$. Delaying the activation gate $d$ led to a maximum alternans magnitude of $37 \mathrm{~ms}$ and a maximum alternans CL range of $65 \mathrm{~ms}$ for delays up to 20 ms. For comparison, we note that previous experimental studies in canine tissue have shown alternans magnitudes of around 50 ms (Gizzi et al., 2013) and alternans CL ranges of around 60$100 \mathrm{~ms}$ (Cherry and Fenton, 2007). When combinations of the gating variables for $I_{\mathrm{Ca}}$ were delayed together with the same delay, the effects of a delay in $f_{C a}$ were dominant when it was included. Introducing a delay in the voltage value used in calculating $K_{1}^{\infty}$ also allowed alternans to be produced with a maximum magnitude of $58 \mathrm{~ms}$. Overall, the minimum delay needed to produce alternans ranged from 1.3 to $9 \mathrm{~ms}$, with smaller minimum values for $K_{1}^{\infty}$ because of the lack of a time constant in its formulation. In addition, because of this difference in formulation, alternans no longer was observed for delays applied to $K_{1}^{\infty}$ that were $2.6 \mathrm{~ms}$ or longer. When gating variables associated with $I_{\mathrm{Kr}}\left(X_{K r}\right), I_{\mathrm{Ks}}\left(X_{K s}\right)$, or $I_{\mathrm{to}}\left(X_{t o}\right.$ and $\left.Y_{t o}\right)$ were delayed, we were not able to produce alternans, most likely because of the resulting significant action potential shortening, which allowed more complete gating variable recovery during the prolonged diastolic intervals.

The delay itself could represent the time needed to sense and react to changes in voltage or intracellular calcium concentration. Channels typically exist in discrete conformational states and 
must transition through several states to change to an open configuration. For example, many channels are tetrameric with each subunit having its own voltage sensor; the sensors in all four subunits must be in the activated position for the channel to be open. Such configurations can be expressed naturally in Markovian channel models (Rudy and Silva, 2006), where the time between peak values of states along a relatively simple closed to open transition can take several ms to tens of ms or even longer, depending on the channel. We expect that our approach may be a useful way to help bridge Markovian and Hodgkin-Huxley formulations phenomenologically; Hodgkin-Huxleytype gating variables with delays potentially could be used as simplified versions of Markovian models with some intermediate states having been replaced with delays. We also note that delays also may be a useful way to incorporate differences in gating timing and dynamics that can occur as a result of disease or mutation (Clancy and Rudy, 2002; Rudy and Silva, 2006).

Implementing delays in multiple gates

We did not examine the results that could occur when delays for multiple gates were combined, except for the gates associated with $I_{\mathrm{Ca}}$. However, it is possible that combining delays in gating variables that could not separately initiate alternans could allow alternans to develop, and this also may depend on the suppression method. For example, because the $I_{\mathrm{Na}}$ current is so brief, we considered that the results could change if the three gating variables were delayed together. However, the delay in producing $I_{\mathrm{Na}}$ with respect to the stimulus current affected action potential formation as well, so that a larger value for the conductance $g_{\mathrm{Na}}$ was necessary. Because doubling $g_{\mathrm{Na}}$ is another alternans suppression method, we combined the increased $g_{\mathrm{Na}}$ with simultaneous delays in $m, h$, and $j$ and found that this combination allowed alternans to be produced by delays of 2-6 ms, as shown in Figs. 8 and 12. However, the maximum alternans magnitude and the range of cycle lengths exhibiting alternans remained smaller than for the original model. The use of delays 
caused slower development of $I_{\mathrm{Na}}$ after the stimulus current finished, which resulted in unusual action potential shapes similar to what can be seen when the stimulus current is close to threshold. The delay in the current effectively increased the threshold current required to generate an action potential; this was partially offset by the increase in $g_{\mathrm{Na}}$. Although the long action potential remained nearly identical in duration to that of the original model at the same cycle length, the short action potential was not nearly as short. The delay slightly increased peak $I_{\mathrm{Na}}$ but otherwise primarily shifted $I_{\mathrm{Na}}$ and each of the gating variables.

A

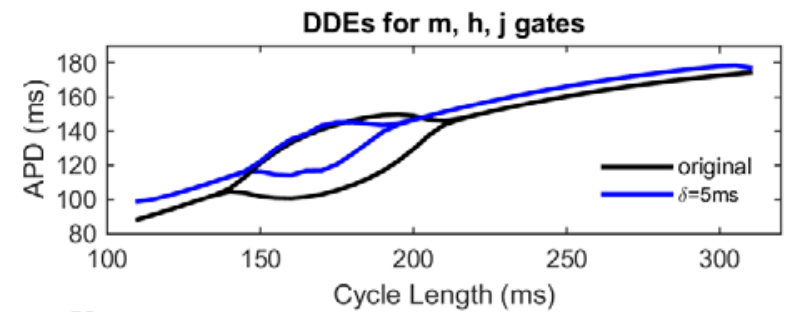

B

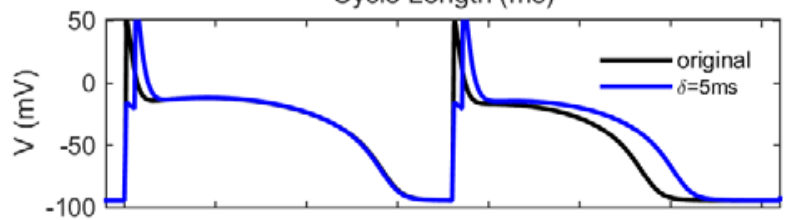

C

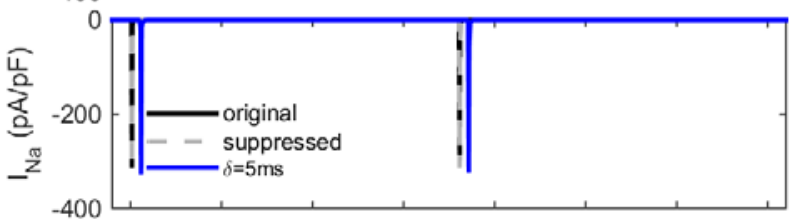

D

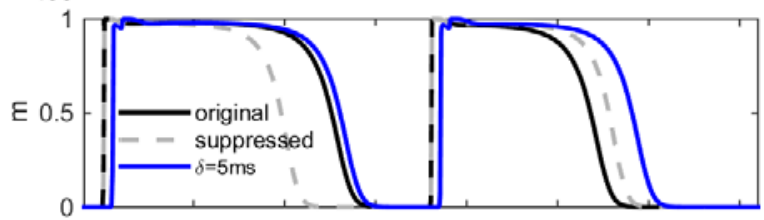

E

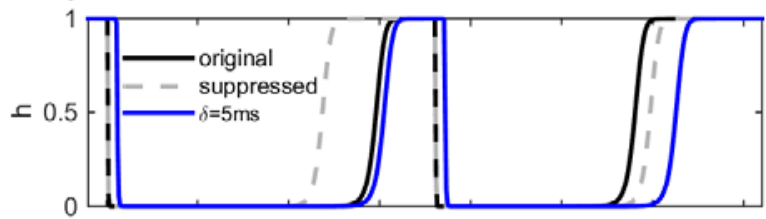

$\mathbf{F}$

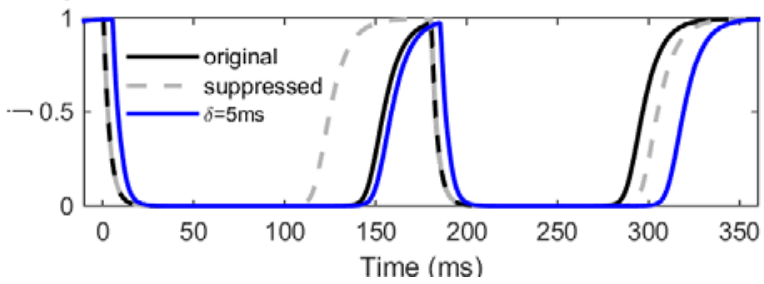


Figure 12. Effects of implementing DDEs simultaneously in the $m, h$, and $j$ gates of the $\mathrm{Na}^{+}$current $I_{\mathrm{Na}}$ using doubled $g_{\mathrm{Na}}$ for suppressing alternans in the original model. A: Bifurcation diagram for cycle lengths of 310 to 110 ms of the original Fox et al. model and after replacing the ODEs for the $m, h$, and $j$ gates with DDEs with delays of 2 and $5 \mathrm{~ms}$. B: Action potentials obtained after $10 \mathrm{~s}$ of pacing at a cycle length of $180 \mathrm{~ms}$ and after adding a 5-ms delay to the $m, h$, and $j$ gates and for the original model. C: $I_{\mathrm{Na}}$ for successive action potentials at a cycle length of $180 \mathrm{~ms}$ for the original, alternans-suppressed, and delayed models. D-F: $m$ gate (D), $h$ gate (E), and $j$ gate (F) for successive action potentials at a cycle length of $180 \mathrm{~ms}$ for the original, alternans-suppressed, and delayed models.

It is possible that other combinations of delayed gating variables, perhaps with different delays across multiple currents, and possibly with different current conductance changes, could allow alternans to be produced in more situations. We tested delaying $X_{\text {to }}$ and $Y_{\text {to }}$ (the two gating variables associated with $I_{t o}$ ) together, but in this case we did not obtain alternans.

It is also possible that delays longer than $20 \mathrm{~ms}$ could lead to alternans formation where shorter delays did not, so that alternans might have been observed with longer delays in some cases. However, longer delays impose an increasing computational burden because the storage of previous values of the membrane potential directly impacts the simulations in terms of memory usage. Applying a delay of $10 \mathrm{~ms}$ in a single cell using a time step of $0.02 \mathrm{~ms}$ would necessitate keeping track of $10 / 0.02=500$ past values of $\mathrm{V}$, requiring 4000 bytes of additional memory if using double precision. In a tissue simulation, that amount would be multiplied by the number of nodes in the discretized domain. In the benchmark problem proposed in (Niederer et al., 2011), the modest-size cuboid tissue would require 1602.2 MB of past tracking memory for a $0.1 \mathrm{~mm}$ discretization. For ventricle simulations the number of nodes may vary between 2.5 and 3.5 million 
(Romero et al., 2010), which would require from 9.3 to 13 GB of additional memory. For these reasons it is clear that delays larger than $20 \mathrm{~ms}$ could compromise the computational performance in large scale simulations. In addition, we suspect such long delays are highly unlikely to be physiologically relevant. Large delay values can change action potential morphology more significantly as the dependence on previous states extends further back in time. Also, in our study, we found large alternans magnitudes at delay values of $20 \mathrm{~ms}$ only for delays applied to the $f$ and $f_{C a}$ gates.

Effects of delays on existing alternans

In our results so far, alternans was always suppressed to ensure that the DDE was responsible for any alternans that was observed. However, when alternans was present with no delay, the addition of a delay could modify characteristics of the alternans that occurred. Figure 13 shows what happens when a delay is added to the $f$ gate without suppressing alternans in the original model. A small delay of 1-2 ms has little effect on the original model, as the magnitude of alternans is within $1 \mathrm{~ms}$ and the onset of alternans is within $5 \mathrm{~ms}$ of the values of the original model. As the delay is increased up to $20 \mathrm{~ms}$, the magnitude of alternans increases, as does the cycle length range of alternans. Therefore, using a DDE can enhance alternans that already exists in a model and thus has the potential to provide a method for increasing the magnitude and CL range of alternans in models that have small alternans magnitudes and CL ranges. 

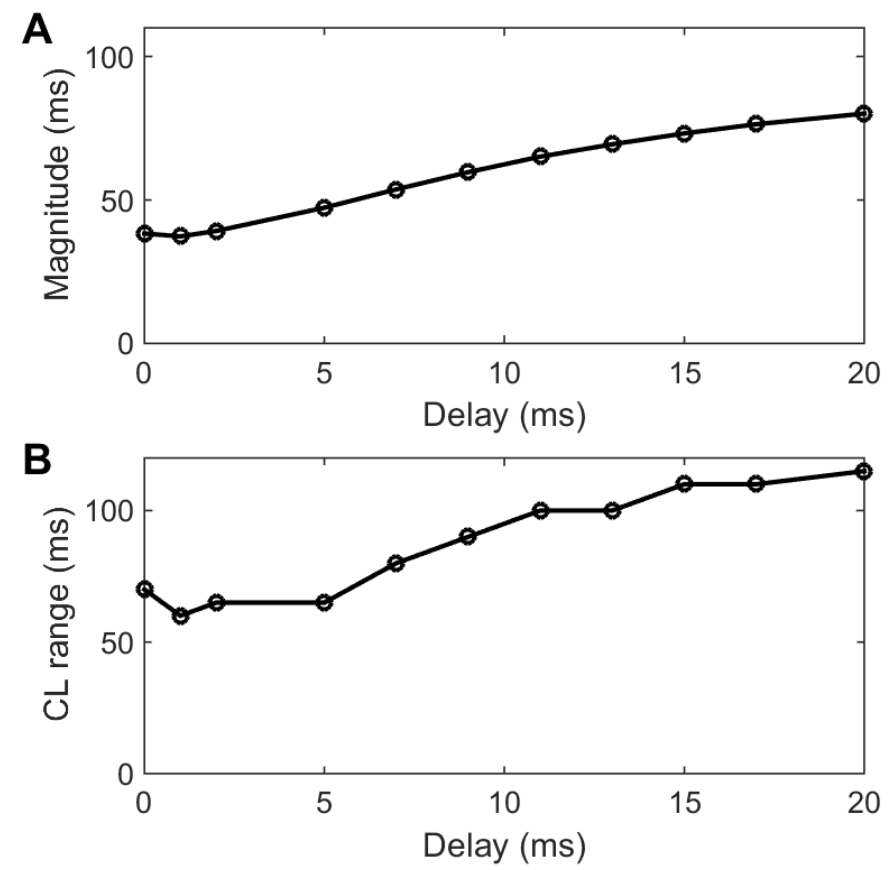

Figure 13. Alternans magnitude (A) and range of cycle lengths exhibiting alternans (B) resulting from a delay in the $f$ gate when alternans was not suppressed in the original model (which corresponded to a delay of $0 \mathrm{~ms}$ ).

Other alternans mechanisms

A number of other mechanisms have been shown previously to give rise to alternans in cardiac cells. Voltage-based mechanisms (Nolasco and Dahlen, 1968) traditionally have been explained by steep APD restitution (Guevara et al., 1984) and it has been shown that they can be enhanced or suppressed by memory (Cherry and Fenton, 2004; Otani and Gilmour, 1997; Tolkacheva et al., 2003). Oscillations in intracellular calcium cycling (Chudin et al., 1999; Eisner et al., 2000; Restrepo and Karma, 2009; Sato et al., 2013; Shiferaw et al., 2003) can arise via nonlinear dependence of calcium release from the sarcoplasmic reticulum (SR) on SR load as well as fluctuations and diffusion of calcium. Many additional mechanisms, such as conduction velocity restitution and action potential shape (Cherry and Fenton, 2004; Echebarria and Karma, 2007, 2002; Mironov et 
al., 2008) as well as spatial heterogeneity involving regions with and without alternans (Bernus et al., 2005; Fenton et al., 2008; Gizzi et al., 2013), can affect alternans formation in tissue. We expect that the mechanism proposed here via DDEs could interact with and possibly enhance other mechanisms and further study will be needed to determine methods for distinguishing among different mechanisms.

\section{Limitations}

In this study, we did not consider tissue dynamics. It is known that intercellular coupling can affect the dynamics of alternans significantly. For example, properties like conduction velocity restitution, wavefront curvature, action potential shape, and memory can lead to the accentuation or suppression of alternans in tissue (Cherry and Fenton, 2004; Comtois and Vinet, 1999; Fenton et al., 2002; Otani and Gilmour, 1997; Wei et al., 2015). How such factors interact with delay differential equations remains an open question.

We have studied only a single model, that of Fox et al. Future studies should use other cardiac electrophysiology models to determine whether delay differential equations can be used more broadly to produce alternans. In addition, it would be interesting to extend our method to add alternans as a dynamical feature to models that do not currently exhibit this behavior. This application would require identifying the currents most likely to contribute to alternans formation from inclusion of one or more DDEs. Another future direction is applying delay differential equations to Markov formulations of currents. It may be possible for the use of DDEs to lead to a reduction in model complexity by allowing transitions through multiple states to be represented by a delay. In addition, the use of delay differential equations in modeling intracellular calcium handling is a promising area for further study (Thompson, 2013). We also would like to study whether DDEs affect controllability of alternans (Dubljevic et al., 2008; Echebarria and Karma, 
2007; Garzón et al., 2011; Krogh-Madsen et al., 2010; Muñoz et al., 2010). Finally, other delay differential equation formulations could be explored.

\section{Acknowledgments}

This work was supported in part by the National Science Foundation through Grants CMMI-

1341128 and DMS-1358583. R.W. dos Santos and J. M. Gomes acknowledge the support provided by CAPES, CNPq and FAPEMIG. We also thank Robert F. Gilmour, Jr. and Ryan Thompson for useful discussions. 


\section{References}

Aliev, R.R., Panfilov, A.V., 1996. A simple two-variable model of cardiac excitation. Chaos Solitons Fractals 7, 293-301. doi:10.1016/0960-0779(95)00089-5

Bernus, O., Zemlin, C.W., Zaritsky, R.M., Mironov, S.F., Pertsov, A.M., 2005. Alternating conduction in the ischaemic border zone as precursor of reentrant arrhythmias: a simulation study. Europace 7 Suppl 2, 93-104. doi:10.1016/j.eupc.2005.03.018

Bocharov, G.A., Rihan, F.A., 2000. Numerical modelling in biosciences using delay differential equations. J. Comput. Appl. Math., Numerical Analysis 2000. Vol. VI: Ordinary Differential Equations and Integral Equations 125, 183-199. doi:10.1016/S0377-0427(00)00468-4

Branco, J.R., Ferreira, J.A., da Silva, P., 2010. Non-Fickian delay reaction-diffusion equations: Theoretical and numerical study. Appl. Numer. Math. 60, 531-549. doi:10.1016/j.apnum.2010.01.003

Bueno-Orovio, A., Cherry, E.M., Fenton, F.H., 2008. Minimal model for human ventricular action potentials in tissue. J. Theor. Biol. 253, 544-60. doi:10.1016/j.jtbi.2008.03.029

Burić, N., Todorović, D., 2003. Dynamics of FitzHugh-Nagumo excitable systems with delayed coupling. Phys. Rev. E 67, 066222. doi:10.1103/PhysRevE.67.066222

Campbell, S.A., 2007. Time Delays in Neural Systems, in: Jirsa, V.K., McIntosh, A.R. (Eds.), Handbook of Brain Connectivity, Understanding Complex Systems. Springer Berlin Heidelberg, pp. 6590.

Cherry, E.M., Fenton, F.H., 2007. A tale of two dogs: analyzing two models of canine ventricular electrophysiology. Am. J. Physiol. Heart Circ. Physiol. 292, H43-55. doi:00955.2006

Cherry, E.M., Fenton, F.H., 2004. Suppression of alternans and conduction blocks despite steep APD restitution: electrotonic, memory, and conduction velocity restitution effects. Am. J. Physiol. Heart Circ. Physiol. 286, H2332-41. doi:14751863 
Christini, D.J., Riccio, M.L., Culianu, C.A., Fox, J.J., Karma, A., Gilmour, Jr., R.F., 2006. Control of electrical alternans in canine cardiac Purkinje fibers. Phys. Rev. Lett. 96, 104101.

Chudin, E., Goldhaber, J., Garfinkel, A., Weiss, J., Kogan, B., 1999. Intracellular Ca(2+) dynamics and the stability of ventricular tachycardia. Biophys. J. 77, 2930-2941. doi:10.1016/S0006$3495(99) 77126-2$

Clancy, C.E., Rudy, Y., 2002. Na(+) channel mutation that causes both Brugada and long-QT syndrome phenotypes: a simulation study of mechanism. Circulation 105, 1208-13.

Comtois, P., Vinet, A., 1999. Curvature effects on activation speed and repolarization in an ionic model of cardiac myocytes. Phys. Rev. E 60, 4619. doi:10.1103/PhysRevE.60.4619

Courtemanche, M., Glass, L., Keener, J.P., 1993. Instabilities of a propagating pulse in a ring of excitable media. Phys. Rev. Lett. 70, 2182-2185.

Courtemanche, M., Keener, J., Glass, L., 1996. A Delay Equation Representation of Pulse Circulation on a Ring in Excitable Media. SIAM J. Appl. Math. 56, 119-142.

doi:10.1137/S0036139993259337

Cytrynbaum, E., Keener, J.P., 2002. Stability conditions for traveling pulse: modifying the restitution hypothesis. Chaos 12, 788-799.

Dubljevic, S., Lin, S.-F., Christofides, P.D., 2008. Studies on feedback control of cardiac alternans. Comput. Chem. Eng., Networked and Complex Systems S.I.Control of Networked and Complex Process Systems 32, 2086-2098. doi:10.1016/j.compchemeng.2007.10.019

Echebarria, B., Karma, A., 2007. Amplitude equation approach to spatiotemporal dynamics of cardiac alternans. Phys. Rev. E Stat. Nonlin. Soft Matter Phys. 76, 051911.

Echebarria, B., Karma, A., 2002. Spatiotemporal control of cardiac alternans. Chaos 12, 923-930.

Eisner, D.A., Choi, H.S., Díaz, M.E., O’Neill, S.C., Trafford, A.W., 2000. Integrative analysis of calcium cycling in cardiac muscle. Circ. Res. 87, 1087-1094. 
Elshrif, M.M., Cherry, E.M., 2014. A quantitative comparison of the behavior of human ventricular cardiac electrophysiology models in tissue. PloS One 9, e84401.

doi:10.1371/journal.pone.0084401

Fenton, F.H., Cherry, E.M., Hastings, H.M., Evans, S.J., 2002. Multiple mechanisms of spiral wave breakup in a model of cardiac electrical activity. Chaos 12, 852-892. doi:10.1063/1.1504242

Fenton, F.H., Cherry, E.M., Kornreich, B.G., 2008. Termination of equine atrial fibrillation by quinidine: an optical mapping study. J. Vet. Cardiol. Off. J. Eur. Soc. Vet. Cardiol. 10, 87-103. doi:10.1016/j.jvc.2008.10.002

Fox, J.J., McHarg, J.L., Gilmour, R.F., 2002. Ionic mechanism of electrical alternans. Am. J. Physiol. Heart Circ. Physiol. 282, H516-30. doi:11788399

Garzón, A., Grigoriev, R.O., Fenton, F.H., 2011. Model-based control of cardiac alternans in Purkinje fibers. Phys. Rev. E 84, 041927.

Garzón, A., Grigoriev, R.O., Fenton, F.H., 2009. Model-based control of cardiac alternans on a ring. Phys. Rev. E 80, 021932.

Gizzi, A., Cherry, E.M., Luther, S., Fenton, F.H., 2013. Effects of pacing site and stimulation history on alternans dynamics and the development of complex spatiotemporal patterns in cardiac tissue. Front. Card. Electrophysiol. 4, 71. doi:10.3389/fphys.2013.00071

Gottwald, G.A., 2008. Bifurcation analysis of a normal form for excitable media: Are stable dynamical alternans on a ring possible? Chaos Interdiscip. J. Nonlinear Sci. 18, 013129. doi:10.1063/1.2890430

Gottwald, G.A., Kramer, L., 2006. A normal form for excitable media. Chaos Interdiscip. J. Nonlinear Sci. 16, 013122. doi:10.1063/1.2168393

Guevara, M.R., Ward, G., Shrier, A., Glass, L., 1984. Electrical alternans and period-doubling bifurcations. Comput. Cardiol. 11, 167-170. 
Hall, G.M., Bahar, S., Gauthier, D.J., 1999. The prevalence of rate-dependent dynamics in cardiac tissue. Phys. Rev. Lett. 82, 2995-2998.

Krogh-Madsen, T., Karma, A., Riccio, M.L., Jordan, P.N., Christini, D.J., Gilmour, R.F., 2010. Off-site control of repolarization alternans in cardiac fibers. Phys. Rev. E Stat. Nonlin. Soft Matter Phys. 81, 011915. doi:10.1103/PhysRevE.81.011915

Krupa, M., Touboul, J.D., 2015. Complex Oscillations in the Delayed FitzHugh-Nagumo Equation. J. Nonlinear Sci. 26, 43-81. doi:10.1007/s00332-015-9268-3

Mironov, S., Jalife, J., Tolkacheva, E.G., 2008. Role of Conduction Velocity Restitution and Short-Term Memory in the Development of Action Potential Duration Alternans in Isolated Rabbit Hearts. Circulation 118, 17-25. doi:10.1161/CIRCULATIONAHA.107.737254

Mitchell, C.C., Schaeffer, D.G., 2003. A two-current model for the dynamics of cardiac membrane. Bull. Math. Biol. 65, 767-793. doi:10.1016/S0092-8240(03)00041-7

Muñoz, L.M., Stockton, J.F., Otani, N.F., 2010. Applications of control theory to the dynamics and propagation of cardiac action potentials. Ann. Biomed. Eng. 38, 2865-2876. doi:10.1007/s10439-010-0037-z

Narayan, S.M., Bode, F., Karasik, P.L., Franz, M.R., 2002. Alternans of atrial action potentials during atrial flutter as a precursor to atrial fibrillation. Circulation 106, 1968-1973.

Niederer, S.A., Kerfoot, E., Benson, A.P., Bernabeu, M.O., Bernus, O., Bradley, C., Cherry, E.M., Clayton, R., Fenton, F.H., Garny, A., Heidenreich, E., Land, S., Maleckar, M., Pathmanathan, P., Plank, G., Rodríguez, J.F., Roy, I., Sachse, F.B., Seemann, G., Skavhaug, O., Smith, N.P., 2011. Verification of cardiac tissue electrophysiology simulators using an N-version benchmark. Philos. Transact. A Math. Phys. Eng. Sci. 369, 4331-4351. doi:10.1098/rsta.2011.0139

Noble, D., 1962. A modification of the Hodgkin--Huxley equations applicable to Purkinje fibre action and pace-maker potentials. J. Physiol. 160, 317-352. 
Nolasco, J.B., Dahlen, R.W., 1968. A graphic method for the study of alternation in cardiac action potentials. J. Appl. Physiol. 25, 191-196.

Otani, N.F., Gilmour, R.F., 1997. Memory models for the electrical properties of local cardiac systems. J. Theor. Biol. 187, 409-436.

Pastore, J.M., Girouard, S.D., Laurita, K.R., Akar, F.G., Rosenbaum, D.S., 1999. Mechanism linking Twave alternans to the genesis of cardiac fibrillation. Circulation 99, 1385-1394.

Rappel, W.J., Fenton, F., Karma, A., 1999. Spatiotemporal control of wave instabilities in cardiac tissue. Phys. Rev. Lett. 83, 456-459.

Restrepo, J.G., Karma, A., 2009. Spatiotemporal intracellular calcium dynamics during cardiac alternans. Chaos Woodbury N 19, 037115. doi:10.1063/1.3207835

Romero, D., Sebastian, R., Bijnens, B.H., Zimmerman, V., Boyle, P.M., Vigmond, E.J., Frangi, A.F., 2010. Effects of the Purkinje system and cardiac geometry on biventricular pacing: A model study. Ann. Biomed. Eng. 38, 1388-1398. doi:10.1007/s10439-010-9926-4

Rudy, Y., Silva, J.R., 2006. Computational biology in the study of cardiac ion channels and cell electrophysiology. Q. Rev. Biophys. 39, 57-116. doi:10.1017/S0033583506004227

Rush, S., Larsen, H., 1978. A practical algorithm for solving dynamic membrane equations. IEEE Trans. Biomed. Eng. 25, 389-392. doi:10.1109/TBME.1978.326270

Sato, D., Bers, D.M., Shiferaw, Y., 2013. Formation of spatially discordant alternans due to fluctuations and diffusion of calcium. PloS One 8, e85365. doi:10.1371/journal.pone.0085365

Shiferaw, Y., Watanabe, M.A., Garfinkel, A., Weiss, J.N., Karma, A., 2003. Model of intracellular calcium cycling in ventricular myocytes. Biophys. J. 85, 3666-3686. doi:10.1016/S0006$3495(03) 74784-5$

Smith, H., 2010. An Introduction to Delay Differential Equations with Applications to the Life Sciences. Springer Science \& Business Media. 
Thompson, R., 2013. Using Delay-Differential Equations for Modeling Calcium Cycling in Cardiac Myocytes. Rochester Institute of Technology, Rochester, NY.

Tolkacheva, E.G., Schaeffer, D.G., Gauthier, D.J., Krassowska, W., 2003. Condition for alternans and stability of the 1:1 response pattern in a "memory" model of paced cardiac dynamics. Phys. Rev. E 67, 031904.

Wei, N., Mori, Y., Tolkacheva, E.G., 2015. The role of short term memory and conduction velocity restitution in alternans formation. J. Theor. Biol. 367, 21-28. doi:10.1016/j.jtbi.2014.11.014 